\title{
Evaluation Method for the Metabolic and the Autonomic Nervous Systems Link Response Using Indirect Calorimetry And Heart Rate Variability M. Cadena ${ }^{1,3}$, O. Infante ${ }^{2}$, E. Ramirez ${ }^{2}$, C. Lerma ${ }^{2}$, B. Escalante ${ }^{3}$, G. Sanchez ${ }^{2}$. \\ ${ }^{1}$ Department of Electrical Engineering, Universidad Autonoma Metropolitaria-Iztapalapa, Mexico. \\ ${ }^{2}$ Department of Electromechanical Instrumentation, Instituto Nacional de Cardiología, Mexico. \\ ${ }^{3}$ Department of Electrical Engineering DEPFI, Universidad Nacional Autonoma de Mexico. Mexico.
}

\begin{abstract}
Several evaluation methods exist to search the link between the Metabolic System (MS) and the Autonomic Nervous System (ANS). Most of them are invasive and they are unable to follow fast adaptable mechanism of the MS and ANS response. In this work we explore a different non-invasive method using Indirect Calorimetry (IC) and Heart Rate Variability (HRV). We used the clino-orthostatism maneuver as ANS oriented stimulus on 7 subjects to see the metabolic response and the set up sensitivity to the experiment. The results agree with the physiology knowledge and with our hypothesis on the stimulus-response paradigm. However, the population, the instrumentation and some physiological hypothesis used in this experiement are still under questioning.

Keywords-Autonomic,Calorimetry, Indirect, Heart, Method, Variability.
\end{abstract}

\section{INTRODUCTION}

The link between the Metabolic System (MS) and the Autonomic Nervous System (ANS) has been searched under different pathophysiological circumstances and with different methodologies, trying to understand complex feedback homeostatic mechanism responses [1]. Obesity development, diabetes, insulin resistance, hyperinsulinemia and other human metabolic abnormalities are frequently associated with the ANS dysfunction activity in the form of cause-effect paradigms [2]. Whole and regional-body measurement methods, like urinary excretion or plasma levels turnover of cathecholamines, isotope dilution and microneurography measurements techniques, have been proposed as the most directly form to observe the ANS response [3]. Recently, Heart Rate Variability (HRV) has been used as an indirect but sensitive and reproducible test to investigate ANS tone over the time [4]. On the other hand, Indirect Calorimetry (IC) is considered the noninvasive golden standard to measure, as on line whole body technique, the catabolic-anabolic MS performance. The IC has been used primarily to evaluate the metabolism on steady-state conditions computing the rest energy expediture and metabolic substrate utilization [5]. New IC measurement systems and different measurement approach have started assessing the energy balance of an experimental subjects submited to a variety of stimulus, searching to understand the adaptability dynamics of the MS behavior [6].

Research on the relationship between metabolic balance regulation and the ANS regulation has contradictory results. Whereas some researchers sustain that a MS alteration causes an ANS tone adaptability others see a physiological similar phenomenon in the backwards direction [7]. In other words, an ANS dysfunction is observed as the major cause for metabolic changes; probably because the link response between both systems has not been well understood and it has not yet been possible to measure which one is predominant and the conditions under they migth switch physiological roles. We suspect that using IC and HRV, as primary sensitive MS-ANS link response laboratory measurement technique, it is possible to develop a method to study the steady and the dynamic balance response on the MS when the cause-effect paradigm is conformed with an oriented ANS non-invasive stimulus.

To test this hypothesis, we design an experiment, combining IC and HRV on human subjects submited to clino-orthostatism maneıver, to explore how sensitive the stimulus is; and how instrumentation dependable the method is.

\section{METHODOLOGY}

\section{A. The Model}

The experiment in this work is based in the IC and HRV measurement setups and classical physiological interpretation. Fig. 1 presents in a schematic form our general hypothesis model, where the energy balance and heart rate dynamics are in stationary condition until it is triggered by the clino-orthostatism test.

We assume a mathematical model for the oxygen consumption ( $\mathrm{VO} 2$ ) and carbon dioxide production (VCO2) measurement procedure, where the equations (1) and (2) give it a random process nature interpretation and this is projected to both IC metabolic extended variables: namely $V O 2(t)$ and $V C O 2(t)$.

$$
\begin{gathered}
V O 2(t) \cong V O 2+v V O 2(t)+r_{1}(t) \\
V C O 2(t) \cong V C O 2+v V C O 2(t)+r_{2}(t)
\end{gathered}
$$

where:

$V O 2(t), V C O 2(t)$ are both modeled by a similar random process (RP)

$\mathrm{VO} 2, \mathrm{VCO} 2$ are the expected value of the respective RP. Therefore, they are constants along stationarity

$v V O 2(t), v V C O 2(t)$ are modeled by the standard deviation of the respective $\mathrm{RP}$ 
GENERAL HYPOTHESIS: REGULATION OF ENERGY BALANCE AND HEART RATE VARIABILITY

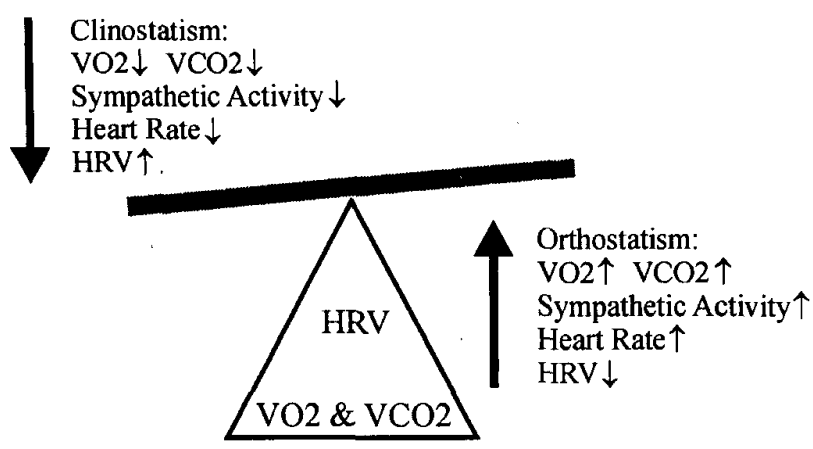

Fig. 1 Physiological model of the metabolic energy balance and AHR dynamics response when an oriented ANS stimulus (lying-to-standing test) is applied to a human subject.

$r_{1}(t), r_{2}(t) \quad$ is a model for the intrinsic instrumentation noise

It is important to observe that $\mathrm{VO} 2$ and $\mathrm{VCO} 2$ are well familiar in the classical IC technique and they are typically used to compute the Resting Energy Expenditure (REE) and also to calculate the Respiratory Quotient $(R Q=V C O 2 / V O 2)$ in order to estimate the metabolic substrate composition (carbohydrates, fat and protein). On the contrary, $v V O 2(t)$ and $\nu V C O 2(t)$, to the best of our knowledge, are new metabolic variables that we suspect are related with the ANS activity and therefore with the HRV.

The nature of $r(t)$ is known in standard IC studies by a limited tolerance variability zone of $\mathrm{VO} 2$ and $\mathrm{VCO} 2$ measurement. Typically this zone is plus-minus one standard deviation of $5 \%$ of the absolute $\mathrm{VO} 2$ and $\mathrm{VCO} 2$ value. However, it is not clear. its behavior under circumstances like the ones we are proposing in this experiment.

\section{B. Experimental Protocol}

The study population was conformed by 7 subjects with different anthropometric and metabolic conditions. The range of the subjects age was between 18 to 45 years old with body mass index from 22 to $45 \mathrm{Kg} / \mathrm{m}^{2}$. All subjects were questioned to avoid a possible abnormal condition on liver, renal, cardiac and endocrine function. None of them had lost weigth or taken medication to change dietary habits during the 3 months proceding the study. All subjects gave informed consent. Each subject was studied during the morning after at least 12 hours of fasting period.

The link between the MS and the ANS was explored by means of the lying-to-standing test (clino-orthostatism maneuver), taking data on a sequence of 15-20 minutes at each stage positions.

The IC and HRV technique were implemented in a set up to take measurements in parallel form at the same time on the same subject. All measurements were made in the same room, which was maintained at a constant temperature, by only one operator.

\section{Measurement Techniques.}

The IC technique was implemented with a standard second generation calorimeter (Life Energy systems Inc, model EMS-50) with mixed gas chamber. Subject expired gas collection was done using a two way valve nondisposable mask with $22 \mathrm{~mm}$ conexion tubing to the calorimeter. The data-log software allowed taking samples every 20 seconds of the $V O 2(\mathrm{ml} / \mathrm{min}), V C O 2(\mathrm{ml} / \mathrm{min}), R Q$, $R E E(\mathrm{Cal} / \mathrm{day})$, tidal volume, respiratory frequency, expired gases temperature and barometric pressure. This instrument computes volumes from BTPS to STPD measurement conditions. The instrument calibration and performance were evaluated, previously to the experiment, using certified gas-mixtures (Scott Specialty Gases) in the physiological range $\left(10,18,21-\mathrm{O}_{2} \%\right.$ and $\left.3,5,10-\mathrm{CO}_{2} \%\right)$ and a Harvard piston type artficial lung, which was able to provide tidal volumes from 0 to $600 \mathrm{ml}$ with different duty-cicles and respiratory frequencies up to 30 per minute.

The IC data was collected in two windows of 10 minutes each, inside of the general 15-20 minutes data logstage in order to assure stationary conditions for both techniques. The IC data windows contain 30 information points each one.

The HRV technique was implemented using an in-house instrument design and manufacture using a commercial off the shelf data acquisition system (National Instruments Inc) interfaced to a standard PC computer. This instrument performance was evaluated following a formal well stablished protocol [8]. The ECG signal was digitalized at $250 \mathrm{~Hz}$ using three standard leads. The time series of RR intervals were computed throughout the five minute standard period and analyzed in consecutive intervals of 1024 data points by the fast fourier transform technique as described [9]. The analytic algorithms were implemented using a commercial software plataform (Lab-View software) according to consensus standards. Two major frequency components were considered in order to compute the "lowfrequency (LF)/ High-frequency (HF)" ratio, well related to the sympathetic-parasympathetic balance activity. The LF components were in the band of 0.04 to $0.15 \mathrm{~Hz}$. Similarly, the HF components were in the band of 0.15 to $0.40 \mathrm{~Hz}$. In addition, SDNN (mean index of the standard deviation of normal-to-normal intervals) and SDSD (standard deviation of differences between adyacent NN intervals) R-R timeserie parameters were calculated together with the LF/HF ratio for statistical analysis.

\section{Data Analysis.}

Two groups of statistical data were conformed, separating clino vs ortho data, on the seven subjects, computing means 
and standard deviations, and then comparison was carried out by the paired " $\mathrm{t}$ " test, one tail, in order to determine whether or not exist significative differences in the clinoortho test. First, statistical analysis was applied to the means using IC and HRV parameters. Second, the same procedure was applied to the standard deviations of the IC parameters. Data are show as means \pm standard error. Differences were considered significant when we found the statistical criteria, $p \leq 0.05$.

\section{RESULTS}

Table I and Table II show respectively the statistical analysis of the means and the standard deviations on the IC and HRV parameters. The heart rate measured by the RR time interval mean was $833 \mathrm{mseg}$ in clino and $771 \mathrm{mseg}$ in ortho position with standard deviations of 121 and 103 respectively. On the other hand, the REE averages were $1361 \mathrm{Cal} /$ day (S.D. $327 \mathrm{Cal} /$ day) in clino compared with $1368 \mathrm{Cal} /$ day (S.D. $400 \mathrm{Cal} / \mathrm{day}$ ) in ortho position.

\section{DIsCUSSION}

\section{A. About the Experiment}

In this experiment, subjects had obesity from moderate to severe degree (BMI, 30 to $45 \mathrm{Kg} / \mathrm{m}^{2}$ ). There is no doubt that the population is poor in variety and number in order to obtain conclusive results. However, this population was selected in order to have a first approach to the measurement set up complexity, sensitive and how tolerant the subjects were to the clino-ortho maneouver

The heart rate was measured in order to have a physiological control variable well stablish and well known in the clino-ortho test.

\section{B. Data Interpretation}

It is apparent that the VO2 and VCO2 did not change during the clino-ortho test, as was expected in the hipothesis. Although, the $R Q$ indeed had a significant variation $(p \leq 0.05)$. Probably because the subjects' catabolism tries to migrate from fat (lypolisis) toward carbohydrates, i.e from 0.78 in clino to 0.84 in ortho. This

TABLE I

STATISTICAL ANALYSIS OF IC \& HRV PARAMETERS IN THE CLINO-ORTHOSTATISM TEST $(n=7)$

\begin{tabular}{|l|c|c|c|c|c|c|}
\hline & $\begin{array}{c}V O 2 \\
\mathrm{~m} / \mathrm{min}\end{array}$ & $\begin{array}{c}V C O 2 \\
\mathrm{ml} / \mathrm{min}\end{array}$ & $R Q$ & SDNN & SDSD & LF/HF \\
\hline Clino & 190 & 150 & 0.78 & 39.3 & 33.7 & 1.59 \\
S.D & \pm 45 & \pm 45 & \pm .05 & \pm 20 & \pm 23 & \pm 1.4 \\
\hline $\begin{array}{l}\text { Ortho } \\
\text { S.D. }\end{array}$ & \pm 91 & 162 & 0.84 & 31.8 & 21.3 & 2.04 \\
\hline "t"test \\
$p$ value
\end{tabular}

TABLE II

STATISTICAL ANALYSIS OF THE STANDARD DEVIATIONS OF THE IC PARAMETERS.

\begin{tabular}{|c|c|c|c|}
\hline & $\begin{array}{c}v V O 2 \\
\mathrm{~m} / \mathrm{min}\end{array}$ & $\begin{array}{c}v V C O 2 \\
\mathrm{ml} / \mathrm{min}\end{array}$ & $R Q$ \\
\hline Clino & $21.3 \pm 22$ & $20.0 \pm 19$ & $0.08 \pm$ \\
\hline Ortho & $38.0 \pm 28$ & $33.7 \pm 23$ & $0.12 \pm$ \\
\hline $\begin{array}{c}\text { "t" test } \\
p \text { value }\end{array}$ & 0.056 & 0.050 & 0.03 \\
\hline
\end{tabular}

result agrees with the marginal SDSD index $(p=0.09)$ and LF/HF ratio $(p=0.14)$ changes indicating a parasympathetic diminished activity, and sympathetic overactivity with the apparent consequences in the MS behaviour via the glucose control mechanism.

The standard deviation analysis reports marginal changes in the $v V O 2(t)(p=0.056)$ and $v V C O 2(t)(p=0.050)$, which might contain some sort of physiological information regarding the link response between the MS and the ANS. The standard deviation from clino $(45 \mathrm{ml} / \mathrm{min})$ to ortho (56 $\mathrm{ml} / \mathrm{min}$ ) makes us think that the instrument was under measurement control. However, before we try to assure anything about the ANS control mechanisms over the MS, we need to characterize $r_{1}(t)$ and $r_{2}(t)$ in order to define a possible signal to noise ratio and separated what is considered information. One possibility for future work is to use a breath-by-breath calorimeter with the feature to obtain samples every two seconds.

The heart rate changed according of what was expected and well documented. In addition, it sustains the physiological model proposed in this experiment regarding the LF/HF values. It is suspected that we did not find relevant variation due to the population $(n=7)$.

Finally, an unexpected result in this experiment is the low IC sensitivity measurements to the postural subject clino-ortho condition. This was observe in the statistical analysis of the respiratory frequency $(16 \pm 7 \mathrm{breath} / \mathrm{min}$ in clino and $17 \pm 5$ in ortho) and the volume minute (VE $=6.4$ \pm 1.9 liters/minute in clino and $7.0 \pm 2.2$ in ortho) respiratory variables.

\section{CONCLUSION}

The hypothesis are partially sustained by this experiment. However, because of the poor number of subjects it is necessary to continue with this work.

The idea to develop a non-invasive method to search the link between the MS and the ANS is very attractive compared with the biochemical invasive methods. These results are relevant because it is observed metabolic changes due to the RQ variation from clino to ortho position with the possibility that this might be related to the ANS activity. The problem is to define whether or not the stimulus is selective for the ANS or we are just observing the cardiovascular response to this test. 
Finally, the experiment puts under test the set up reliability and sensitivity. Probably, because the physiological information is so deep that it is necessary to think in a research oriented technology.

\section{ACKNOWLEDGMENT}

We wish to thank to the personal of the Electromechanical Department of the INC for the permanent support to achive the goals of this project.

\section{REFERENCES}

[1] Manzella, D. et al. "Role of Free Fatty Acids on Cardiac Autonomic Nervous. System in Noninsulin-Dependent Diabetic Patients: Effects of Metabolic Control. Journal of clinical Endocrinology \& Metabolism, Vol. 86,No. 6, 2769-2774, 2001.

[2] Emdin,M. et al. "Hyperinsulinemia and Autonomic Nervous System Dysfunction in Obesity", Circulation. 103:513-519, 2001.

[3] Young JB,Macdonald IA. "Sympathoadrenal activity in human obesity: heterogeneity of findings since 1980 . Int. J. Obes. Relat. Metab. Disord. 16, 959-967.1992.

[4] Hung-Wen Chiu and Tsair Kao. "A Mathematical Model for Autonomic Control of Heart Rate Variation". IEEE-EMBS Magazine, p-p 69-76 March-April,2001.

[5] McClave,S. et al. "Use of Indirect Calorimetry in Clinical Nutrition" Invited Review. NCP, vol. 7, No5,October-1992.

[6] Williams,T.D. et al. "Cardiovascular response to caloric restriction and thermoneutrality in C57BL/6J mice". Am. J. Physiol. Regul. Integr. Comp. Physiol. 282: 1459-1467, May 2002.

[7] Valensi, P. et al. "Glucose-induced thermogenesis, inhibitation of lipid oxidation rate and autonomic dysfunction in non-diabetic obese women". Int. J. Obes. Relat. Metab. Disord. 22(6): 494499. Jun 1998.

[8] Lerma, C. Infante, O. " A system for analysis of Heart Rate variability". Proceeding of ELECTRO-2000, Institute of Chihuahua, Mexico. p-p 63-67.2000.

[9] Special report: "HRV:Standards of Measurement, Physiological Interpretation, and Clinical Use. Circulation, 93: 1043-1065. 1996. 